\title{
Age-Associated Changes in Atrophy of the Extensor Digitorum Longus Muscle in Hindlimb-Suspended Rats
}

J. Phys. Ther. Sci.

20: $129-133,2008$

\author{
MASAmi YoKOGAwa, RPT, BA, MSc, PhD ${ }^{1)}$, TOSHIAKI YAMAZAKI, RPT, BA, MSc, PhD ${ }^{1)}$, \\ KATSUMI INOUE, BSc, MSc ${ }^{1)}$, PleIADES TIHARU INAOKA, RPT, BSc, MHSc ${ }^{2}$, \\ KEIICHI TSUJ, RPT, BA, MHSc${ }^{2)}$, KATSUHIKO TACHINO, MD, DMSc ${ }^{1)}$ \\ 1)Division of Rehabilitation Science, Department of Health Sciences, Graduate School for \\ Health Studies, The University of Kanazawa: 11-80 Kodatsuno 5, Kanazawa, Ishikawa \\ 920-0942, Japan. TEL +81 76-265-2618, FAX +81 76-234-4372, \\ E-mail: yokogawa@mhs.mp.kanazawa-u.ac.jp \\ 2)Ph.D. Candidate, Graduate School for Health Studies, The University of Kanazawa
}

\begin{abstract}
Purpose] An experiment was carried out to investigate possible age-associated changes in the cross-sectional area of the type II fibers of the extensor digitorum longus (EDL) of male rats during hindlimb unweighting. [Subjects] Nine 3-month-old and seven 8-month-old rats were divided into three groups each. [Methods] One group was reared under hindlimb suspension for one week, another group for two weeks and a control group under normal rearing conditions for three weeks. The cross-sectional area of 200 to 230 muscle fibers of the right EDL from each rat was measured, followed by random sampling of 15 type II fibers from each of the six groups that were used for a comparison of their mean values. In addition, 50 type II fibers were selected for a comparison of their frequency distribution patterns. [Results] Significant atrophic changes were demonstrated in EDL of the 3-month-old rats in hindlimb suspension for one week in comparison to the control group, but the 8-month-old rats showed no significant atrophic changes in any of the groups. [Conclusion] The atrophying process of the rat EDL may differ depending on its age, and this process in aged skeletal muscle fibers may be delayed for a short period of time during unweighting.
\end{abstract}

Key words: Unweighting, Aging, Extensor digitorum longus

(This article was submitted Nov. 26, 2007, and was accepted Feb. 1, 2008)

\section{INTRODUCTION}

Various changes in the physiological responses of the body occur with aging. In the skeletal muscle of the elderly there is a decrease in the muscle mass as well as a decline in muscle strength ${ }^{1)}$. Atrophy of muscles occurs due to prolonged inactivity that may be associated with a disease or injury. The atrophying process in the absence of activity in the elderly may differ from that in the young due to ageassociated changes in their skeletal muscles. Inquiry into the effects of inactivity on aged skeletal muscles may be of importance to enhance efficacy of physical therapy for the elderly.

Hindlimb suspension of rats has been used to create systemic disuse atrophy, in which the hindlimbs are unweighted during the experimental period. The muscle atrophy occurring during prolonged unweighting is known to be muscle- and/ or fiber-type-specific. There is supporting evidence to show that the extensor digitorum longus (EDL), a non-postural muscle, becomes less atrophied compared to the soleus muscle, a postural muscle, during prolonged unweighting ${ }^{2}$. In addition, type 
II muscle fibers have been found to be less affected by prolonged unweighting than those of type $\mathrm{I}^{3,4)}$.

Past studies on the effects of aging on the responses of skeletal muscles to prolonged unweighting have produced conflicting results: similar responses among old and mature rat skeletal muscles ${ }^{5}$; or more marked changes in the contractility in the older animals than in the younger animals ${ }^{6}$; and morphologically or histochemically more marked atrophy in young than in aged rats ${ }^{7,8)}$. In a past study, we investigated changes in the cross-sectional area of the rat soleus muscle fibers during hindlimb suspension and observed differences in the cross-sectional area of both type I and II fibers between aged and young rats ${ }^{9}$. However, type II fibers account for only about $20 \%$ of the total amount of muscle fibers of the soleus muscle. Considering the fact that type II fibers predominantly atrophy with age ${ }^{1)}$, we considered that examination of a muscle sample with a larger number of type II fibers would be of significance. Accordingly, the purpose of this study was to determine whether changes in the cross-sectional area of type II fibers of the rat EDL during prolonged unweighting would differ with their age.

EDL is a fast muscle in mature rats and is composed of more than $95 \%$ type II fibers ${ }^{10)}$. Therefore, two hypotheses were presented for the current study as follows: 1) the atrophying process of EDL of the rats would differ according to their age; and 2) the progression of EDL atrophy would be slower in older rats.

\section{METHODS}

The animals used for the experiment were nine male Fischer 344 rats (Charles River Japan, Atsugi, Japan) aged three months old with a body weight ranging from 210 to $246 \mathrm{~g}$ and seven Fischer 344 rats aged eight months old with a body weight ranging from 350 to $400 \mathrm{~g}$. The experiment was commenced following a 1 -week period of preliminary rearing of all of the 16 rats. The two age groups were divided into three groups each, one group of which consisted of rats reared under hindlimb suspension for one week (HS1), another for two weeks (HS2) and a control group under normal rearing conditions for three weeks. The number of rats assigned to each group was as follows: three 3-month-old rats to each of the 3month-old groups, and two 8-month-old rats to each of HS1 and HS2, and the remaining three rats to the control group. All of the rats were weighed before and after the experiment.

The life span of rats is two to three years ${ }^{10)}$. Differentiation of the rat's EDL becomes complete after about 20 days ${ }^{11)}$. Among the hindlimb muscles of the rat, the mass and circumference of the plantar muscle develop rapidly until the age of 10 weeks, and thereafter the growth plateaus, but continues to develop slightly up to 50 weeks ${ }^{12)}$. After 30 weeks of age, EDL begins to show a variety of changes occurring in its mass and circumference, and the development of aging symptoms, and individual differences become greater ${ }^{12}$. In the current study, the 3 -month-old rats were considered to have completion of EDL differentiation, while the 8month-old rats were considered to have early signs of aging in their hindlimbs.

Each rat was reared in its own individual cage $(280 \times 440 \times 180 \mathrm{~mm})$ at a room temperature of 21 to $26^{\circ} \mathrm{C}$ under 12-hour light/dark cycles with the light hours lasting from 0800 to 2000 hours. This study was carried out with the approval of the Committee for Laboratory Animal Experiments, Takaramachi Campus, Kanazawa University, and the rats were cared for in accordance with the Guidelines for Animal Experiments prepared by the same committee (Approval No. 031671).

At the commencement of the experiment, in the hindlimb-suspension groups, a jacket was applied to each rat under anesthesia with diethyl ether. The jacket application and construction of the cage are described in detail elsewhere ${ }^{13}$. On application of the jacket the hindlimbs of the rat were immediately freed from weight bearing and, by the use of the forelimbs, they were free to move in an anteroposterior direction along the long axis of the cage as well as to be able to rotate 360 degrees. Food and water were given ad libitum during the entire experimental period.

The right EDL was selected for examination and was excised from each rat under anesthesia with sodium pentobarbital (50 mg/kg weight) on completion of the experiment. The wet weight of each EDL was measured immediately after isolation, followed by excision of a $5-\mathrm{mm}$ section from the center of the muscle belly, which was then fixed to a piece of cork. The fixed muscle sections were instantly frozen in isopentane that was cooled with liquid nitrogen and then stored at $-70^{\circ} \mathrm{C}$ until they were analyzed. 
Frozen sections were further cut into $10-\mu \mathrm{m}$ on a cryostat at a temperature of $-25^{\circ} \mathrm{C}$ and stained with ATPase $^{14)}$ at a $\mathrm{pH}$ of 10.6. The sections were observed under a light microscope (Olympus, BX50), photographed using a digital camera, and the information was entered into a computer. The section images in the computer were classified into type I and II fibers using NIH image 1.62. Each of the cross-sectional areas of 200 to 230 type I and II fibers were measured. Table 1 shows the percentage distribution of the EDL fiber types.

As shown in Table 1, type II fibers accounted for more than $95 \%$ of the rat EDL's composition, with the number of type I fibers being very small. Therefore, statistical analysis focused only on type II fibers in this study. A wide range in the distribution of cross-sectional area of the fibers was seen, which would have resulted in a biased view of the change in the difference in frequency distribution patterns if a comparison of only the mean values had been made. Therefore, analysis was made by comparison of both the mean values and the frequency distribution patterns of type II fibers ${ }^{9)}$ in order to clarify the characteristics of the changes in their cross-sectional areas from multiple aspects.

Fifteen type II fibers were randomly selected from each group to compare the mean value of their cross-sectional areas, and two-way analysis of variance was performed using age and suspension period as factors. When the interaction between age and suspension period was not statistically significant, a multiple comparison was made for each age group using Bonferroni's method.

Fifty type II fibers were randomly selected from each group, and their key statistics were calculated. The differences in the frequency distribution patterns of the cross-sectional areas between and within age groups were analyzed using Kolmogorov-Smirnov's two-sample test followed by Bonferroni's correction method for multiple comparison. A significant difference between the two age groups using this test would be regarded as showing a difference in their probability distributions in the population. This was followed by analysis of the differences in frequency distribution patterns due to the presence of a significant difference between the two age groups based on the key statistics.

An alpha level of 0.05 was selected for statistical significance in the current study, and the Statistics Package for Social Sciences version 11.0 (SPSS
Table 1. Percentage distribution of types I and II fibers of the extensor digitorum longus

\begin{tabular}{llcc}
\hline & & Type I fibers (\%) & Type II fibers (\%) \\
\hline 3-month-old & Control & $3.8 \pm 0.8$ & $96.2 \pm 0.8$ \\
& HS1 & $3.4 \pm 0.3$ & $96.6 \pm 0.3$ \\
& HS2 & $3.2 \pm 1.2$ & $96.8 \pm 1.2$ \\
8-month-old & Control & $4.2 \pm 3.6$ & $95.8 \pm 3.6$ \\
& HS1 & $1.9 \pm 2.6$ & $98.1 \pm 2.6$ \\
& HS2 & $4.5 \pm 2.4$ & $95.5 \pm 2.4$ \\
\hline
\end{tabular}

Values are mean \pm standard deviation.

HS1: hindlimb suspension for one week;

HS2: hindlimb suspension for two weeks.

Japan Inc.) was used for the data analysis.

\section{RESULTS}

There was no interaction effect in the mean crosssectional areas among the groups $(\mathrm{p}=0.054)$. A multiple comparison revealed a significant decrease in the mean cross-sectional area of the HS2 group of the 3-month-old rats compared with the control group $(\mathrm{p}<0.05$; Table 2$)$. No significant differences were observed among the groups of the 8-monthold rats.

The frequency distribution patterns in the HS1 and HS2 groups showed significant differences between the two age groups $(p<0.001)$, but those of the control groups did not.

The frequency distribution patterns for the 3month old rats were significantly different between the HS2 and control groups as well as between the HS1 and HS2 groups $(p<0.01$; Table 3$)$. The frequency distribution patterns for the 8-month-old rats were significantly different between the HS1 and control groups as well as between the HS1 and HS2 groups ( $p<0.01$ and $p<0.05$, respectively; Table $3)$. Based on the key statistics for each group, the mean values and the range of frequency distribution for the 3-month-old rats were largest in the control group, followed by the HS1 and HS2 groups in decreasing order. For those of the 8-month-old rats, they were the largest in the HS1 group, followed by the HS2 and control groups (Table 4).

\section{DISCUSSION}

Although the synergistic effects of age and suspension period were not apparent in this experiment, the frequency distribution patterns 
Table 2. Comparison of the mean cross-sectional area of type II fibers of the extensor digitorum longus

\begin{tabular}{lcccccc}
\hline & $\begin{array}{c}\text { Control } \\
\text { group }\end{array}$ & $\begin{array}{c}\text { HS1 } \\
\text { group }\end{array}$ & $\begin{array}{c}\text { HS2 } \\
\text { group }\end{array}$ & Age & Suspension period & Interaction \\
\hline 3-month-old & $2046 \pm 1164$ & $1500 \pm 485$ & $1138 \pm 319^{*}$ & $\mathrm{p}<0.001$ & $\mathrm{p}=0.068$ & $\mathrm{p}=0.054$ \\
8-month-old & $2248 \pm 869$ & $2660 \pm 989$ & $2215 \pm 880$ & & & \\
\hline
\end{tabular}

Unit: $\mu \mathrm{m}^{2}$, Values are mean \pm standard deviation. HS1: hindlimb suspension for one week; HS2: hindlimb suspension for two weeks. ANOVA: analysis of variance.

*: $\mathrm{p}<0.05$ (vs. Control).

Table 3. Comparison of the frequency distribution patterns for each age group

\begin{tabular}{|c|c|c|c|c|c|c|}
\hline & \multicolumn{3}{|c|}{ 3-month-old } & \multicolumn{3}{|c|}{ 8-month-old } \\
\hline & Control & HS1 & HS2 & Control & HS1 & HS2 \\
\hline Control & --- & NS & $* *$ & --- & $* *$ & NS \\
\hline HS1 & --- & --- & $* *$ & --- & --- & $*$ \\
\hline
\end{tabular}

HS1: hindlimb suspension for one week; HS2: hindlimb suspension for two weeks.

$* *: \mathrm{p}<0.01, *: \mathrm{p}<0.05, \mathrm{NS}$ : no significant difference.

Table 4. Key statistics showing the frequency distribution patterns of type II fibers

\begin{tabular}{lrrrrrrrr}
\hline & \multicolumn{3}{c}{3 3-month-old } & & \multicolumn{3}{c}{ 8-month-old } \\
\cline { 2 - 4 } \cline { 7 - 8 } & Control & HS1 & HS2 & & Control & HS1 & HS2 \\
\hline Mean & 1818 & 1677 & 1286 & & 1882 & 2680 & 2166 \\
SD & 815 & 600 & 350 & & 792 & 1021 & 941 \\
Minimum & 718 & 842 & 756 & & 437 & 900 & 895 \\
Maximum & 4203 & 3283 & 2327 & & 3441 & 4565 & 4267 \\
Range & 3485 & 2441 & 1571 & & 3004 & 3666 & 3372 \\
\hline
\end{tabular}

Unit: $\mu \mathrm{m}^{2}$, Values are mean \pm standard deviation.

HS1: hindlimb suspension for one week; HS2: hindlimb suspension for two weeks. SD: standard deviation.

revealed differences between the age groups. Changes in the frequency distribution patterns based on the suspension period were examined according to the results presented in Table 4. For the 3-monthold rats, the mean value and the range of the frequency distribution patterns were small in the two suspension groups compared with those of the control group, whereas for the 8-month-old rats they were large. In other words, the EDL fibers in the HS2 group of the 3-month-old rats showed significant atrophic changes, but in the HS1 group they were non-significant. Furthermore, atrophic changes may have occurred in the two suspension groups of the 8-month-old rats, but at the same time, they appeared to have maintained either their existing cross-sectional area or hypertrophied fibers.
When the frequency distribution patterns of the cross-sectional areas were examined vis-á-vis the presence or absence of atrophy, it can be said that no significant atrophy occurred in the HS1 group of both the 3-and 8-month-old rats, but significant atrophy was seen to occur in the HS2 group of the 3month-old rats. In our past study, using the same method as that of the current study, we examined the changes in the cross-sectional areas of the rat soleus muscle after unweighting at 1,2, and 4 weeks (HS1, HS2 and HS4, respectively) ${ }^{9}$. In type I fibers, which constitute the bulk of the rat soleus muscle fibers, the frequency distribution patterns of the cross-sectional areas showed significant changes compared with those of the control groups for the HS1 group of the 3-month-old rats and HS2 group of the 8-month-old rats. The results of the two studies suggest that the amount of atrophy may be less in EDL than the soleus muscle after similar unweighting periods. This is consistent with the generally accepted view that the degree of atrophy during unweighting of a non-postural muscle such as EDL is smaller than in a postural muscle such as the soleus muscle ${ }^{2}$.

In the current study, the frequency distribution patterns for the 8-month-old rats showed no significant change when compared with the control group even after two weeks of hindlimb suspension, suggesting slower atrophic change in the 8-monthold rats than in the 3-month-old rats. Aged skeletal muscle has an attenuated ability to alter its size in response to inactivity ${ }^{15)}$. Such age-associated changes in the skeletal muscle responses observed in this study may have been responsible for the difference seen in the process of the changing crosssectional areas between the two age groups. It is possible that rats aged 8 months may develop atrophic changes with prolongation of the unweighting period.

Gehrke et al. ${ }^{4)}$ carried out an 18-day hindlimb suspension in rats aged 30 months and observed a 
significant decrease in the cross-sectional area of EDL type II fibers in the suspension group compared with the control group without significant decrease in that of type I fibers. This suggests that the responses of EDL type II fibers to unweighting may change with growth and aging. Comparison of the mean cross-sectional areas yielded no interaction (Table 2), suggesting that age and suspension period are independent factors that have an additive action. In other words, the result seen for type II fibers of EDL of the 8-month-old rats suggests that there is an increase in the crosssectional area when the hindlimbs are in suspension for up to two weeks. Type II fibers of EDL have been reported to show both presence ${ }^{4)}$ and absence $^{16)}$ of a significant decrease in crosssectional area after unweighting. In the soleus muscle, the rate of protein degradation increases compared with its synthesis during the unweighting period, but there is, on the whole, a large protein loss after 10 days to two weeks of unweighting ${ }^{17)}$. EDL contains a large percentage of type II fibers and becomes stretched during unweighting. The time course of changes in protein synthesis during unweighting may differ between the soleus muscle and EDL, and so also may the sensitivity to stretching, depending on the age of the rats. In addition, since the mean value, in this study, by analysis of variance was on the borderline of significance $(\mathrm{p}=0.054)$, the synergic effects of age and suspension period cannot be ruled out completely. It is possible that aging as well as other factors may have caused a transient increase in the cross-sectional area of the EDL during unweighting. However, it is not certain why the cross-sectional area increased in the relatively early stage of unweighting in the 8-month-old rats. Further studies are required because only a small number of rats were used in this study.

\section{ACKNOWLEDGEMENTS}

The authors thank Prof. Shimpachiro Ogiwara, RPT, SRP(UK), ONC(UK), MCPA(C), BPT(C), MA(USA), PhD(USA) and Mrs. Sandra M. Ogiwara, CSP(UK), HT(UK), CPA(C), BScPT(C), for their review and advice on the manuscript.

\section{REFERENCES}

1) Brooks SV, Faulkner JA: Skeletal muscle weakness in old age: underlying mechanisms. Med Sci Sports
Exerc, 1994, 26: 432-439.

2) Jaspers SR, Tischler ME: Atrophy and growth failure of rat hindlimb muscles in tail-cast suspension. J Appl Physiol, 1984, 57: 1472-1479.

3) Thompson LV: Skeletal muscle adaptations with age, inactivity, and therapeutic exercise. J Orthop Sports Phys Ther, 2002, 32: 44-57.

4) Gehrke AG, Krull MS, McDonald RS, et al.: The effect of non-weight bearing on skeletal muscle in older rats: an interrupted bout versus an uninterrupted bout. Biol Res Nurs, 2004, 5: 195-202.

5) Stump CS, Tipton CM, Henriksen EJ: Muscle adaptations to hindlimb suspension in mature and old Fischer 344 rats. J Appl Physiol, 1997, 82: 1875-1881.

6) Thompson LV, Johnson SA, Shoeman JA: Single soleus muscle fiber function after hindlimb unweighting in adult and aged rats. J Appl Physiol, 1998, 84: 1937-1942.

7) Brown M, Hasser EM: Differential effects of reduced muscle use (hindlimb unweighting) on skeletal muscle with aging. Aging Clin Exp Res, 1996, 8: 99-105.

8) Simard C, Lacaille M, Vallieres J: Effects of hypokinesia/hypodynamia on contractile and histochemical properties of young and old rat soleus muscle. Exp Neurol, 1987, 97: 106-114.

9) Yokogawa $M$, Inoue $K$, Yamazaki $T$, et al.: Agerelated differences in the atrophy for the rat soleus muscle subjected to hindlimb suspension: comparison of the pattern of frequency distributions of muscle fiber cross-sectional area. J Tsuruma Health Sci Soc, 2007, 31: 71-80 (in Japanese).

10) Japanese Society for Laboratory Animals (ed): Scientific Basis for Experimental Research on Animals -Technical Edition-. Tokyo: Maruzen, 1991, pp118-127 (in Japanese).

11) Okada $S$, Nonaka I, Ishiura $S$, et al.: A histochemical study of muscle fiber differentiation in the rat muscle. Shinkeinaika, 1981, 15: 363-370 (in Japanese).

12) Tamaki $T$, Uchiyama $S$ : Absolute and relative growth of rat skeletal muscle. Physiol Behav, 1995, 57: 913919.

13) Yamazaki $T$ : Influence of hindlimb unweighting and intermittent weight bearing on dynamics of nuclei in rat soleus muscle. J Jpn Phys Ther Assoc, 2003, 6: 1-6.

14) Nonaka I: Muscle Pathology for the Clinician, 3rd ed. Tokyo: Japan Medical Journal Co, 1999, pp2-14 (in Japanese).

15) Husom AD, Ferrington DA, Thompson LV: Agerelated differences in the adaptive potential of type I skeletal muscle fibers. Exp Gerontol, 2005, 40: 227235 .

16) Desplanches D, Mayet $\mathrm{MH}$, Sempore B, et al.: Structural and functional responses to prolonged hindlimb suspension in rat muscle. J Appl Physiol, 1987, 63: 558-563.

17) Thomason DB, Booth EW: Atrophy of the soleus muscle by hindlimb unweighting. J Appl Physiol, 1990, 68: 1-12. 